\title{
MG132 selectively upregulates MICB through the DNA damage response pathway in A549 cells
}

\author{
DAN LUO $^{1 *}$, XI-WEN DONG ${ }^{2,3^{*}}$, BING YAN $^{4 *}$, MEI LIU ${ }^{4}$, TIAN-HUI XUE ${ }^{4}$, HUI LIU $^{4}$, \\ JUN-HAO YOU ${ }^{4}$, FANG LI ${ }^{4}$, ZI-LING WANG ${ }^{1}$ and ZHI-NAN CHEN ${ }^{1}$ \\ ${ }^{1}$ College of Life Science and Bioengineering, School of Science, Beijing Jiaotong University, Beijing 100044; \\ ${ }^{2}$ Department of Experimental Hematology, Beijing Institute of Radiation Medicine; \\ ${ }^{3}$ Beijing Key Laboratory for Radiobiology, Beijing 100850; ${ }^{4}$ Department of Oncology, \\ Hainan Branch of General Hospital of PLA, Sanya, Hainan 572013, P.R. China
}

Received June 6, 2018; Accepted October 23, 2018

DOI: $10.3892 / \mathrm{mmr} .2018 .9676$

\begin{abstract}
Natural killer (NK) cells recognize stress-activated NK group 2, member D (NKG2D) ligands in tumors. In the present study, the expression levels of NKG2D ligands were examined in four lung cancer cell lines (A549, PLA801D, NCI-H157 and NCI-H520). In the A549 cells, the expression of MHC class I polypeptiderelated sequence (MIC)A/B and UL16 binding protein (ULBP)1 was weak, the expression of ULBP2 was typical, and neither ULBP3 nor ULBP4 were expressed. The mechanism underlying the regulatory effect of a cancer treatment agent on the expression of NKG2D ligands was investigated using the proteasome inhibitor MG132. Following treatment for $8 \mathrm{~h}$ with MG132, the transcription levels of MICB and ULBP1 were upregulated 10.62- and 11.09-fold, respectively, and the expression levels of MICB and ULBP1 were increased by 68.18 and $23.65 \%$, respectively. Notably, MICB exhibited significant time-dependent change. MG132 increased the transcription of MICB by acting at a site in the 480-bp MICB upstream promoter. The activity of the MICB
\end{abstract}

Correspondence to: Dr Zhi-Nan Chen or Dr Zi-Ling Wang, College of Life Science and Bioengineering, School of Science, Beijing Jiaotong University, 3 Shangyuan Cun, Haidian, Beijing 100044, P.R. China

E-mail: znchen@fmmu.edu.cn

E-mail: zlw@bjtu.edu.cn

*Contributed equally

Abbreviations: ATM, ataxia telangiectasia mutated; ATR, ATM-Rad3-related; mAb, monoclonal antibody; MICA, MHC class I polypeptiderelated sequence A; MICB, MHC class I polypeptiderelated sequence $B$; NK, natural killer; NKG2D, NK group 2, member D; NSCLC, non-small cell lung cancer; RT-qPCR, reverse transcription-quantitative polymerase chain reaction; ULBP, UL16 binding protein

Key words: MG132, MHC class I polypeptiderelated sequence B, natural killer cells, immunotherapy, chemotherapy promoter was upregulated 1.77-fold following treatment with MG132. MG132 treatment improved the cytotoxicity of NK cells, which was partially blocked by an antibody targeting NKG2D, and more specifically the MICB molecule. The expression of MICB induced by MG132 was inhibited by KU-55933 [ataxia telangiectasia mutated (ATM) kinase inhibitor], wortmannin (phosphoinositide 3 kinase inhibitor) and caffeine (ATM/ATM-Rad3-related inhibitor). The phosphorylation of checkpoint kinase 2 (Chk2), an event associated with DNA damage, was observed following treatment with MG132. These results indicated that MG132 selectively upregulates the expression of MICB in A549 cells, and increases the NKG2D-mediated cytotoxicity of NK cells. The regulatory effect of MG132 may be associated with the activation of Chk2, an event associated with DNA damage. The combination of MG132 with NK cell immunotherapy may have a synergistic effect that improves the therapeutic effect of lung cancer treatment.

\section{Introduction}

Human natural killer (NK) cells are a subpopulation of lymphocytes that are important in innate immunity, adaptive immunity and reproduction (1). NK cells are major effector cells of the innate immune system, being important in tumor immunity, antiviral infection and the removal of 'non-self' cells (2-4). The T cell receptor is a specific marker on the surface of all T cells which recognizes specific antigens and exerts an immunological function. The difference between $\mathrm{T}$ cells and NK cells is that the latter are not dependent on the MHC-I molecules of tumor cells to initiate a toxic effect and their activity is the result of the interaction of various receptor molecules with corresponding ligands $(5,6)$. The interaction between the NK cell lectin-like receptor, NK group 2, member $\mathrm{D}$ (NKG2D), and NKG2D ligands has been demonstrated to be important in targeted cell killing by NK cells (5).

NKG2D is an activation receptor $(7,8)$ and is expressed on a variety of immune cells $(5,9)$. NK cells are heterogeneous cells and those expressing the NKG2D receptor may be important in targeting and eliminating malignant cells. There are two categories of NKG2D ligands: MHC class I polypeptiderelated sequence (MIC)A and B, and UL16 binding protein 
(ULBP)1-5 (10). NKG2D ligands are expressed on the surface of tumor cells and MICs are induced cell surface antigens $(11,12)$. Previous studies have revealed that numerous factors, including cell stress, heat shock, infection, DNA damage and transformation, can upregulate the expression of MICs (13-18).

Previous studies have demonstrated that specific MHC-restricted cytotoxic $\mathrm{T}$ cells cannot function when MHC-I molecules on tumor cells are lost or vary from the norm. In this case, the NKG2D-NKG2D ligand signaling pathway is important in antitumor immunity, with NKG2D as the main activating receptor of NK cells that induces anti-tumor effects $(19,20)$. Following the interaction between NKG2D and corresponding induced ligands on target cells, NKG2D binds to an adaptor protein and transmits an activated signal to NK cells. In this way, NK cells obtain the ability to lyse the target cells (21). In addition, $\mathrm{CD} 8^{+} \mathrm{T}$ cells provide the necessary synergistic stimulating signals, and the activation of the NKG2D pathway largely determines the intensity of the cell's immune response against cancer.

At present, NK cell-based immunotherapy combined with chemotherapy is widely used clinically; however, the mechanism by which chemotherapy regulates the antitumor activity of NK cells, particularly NKG2D-mediated cell death, remains to be fully elucidated to the best of our knowledge. Although the coding regions of NKG2D ligands are conservative, the homology of the 5' non-translated region is low, which indicates that the regulation of their expression may be specific to different stimuli or lesions.

Therefore, the A549 cell line was used as the cell model in the present study in order to investigate the regulatory effect of a cancer treatment agent (the proteasome inhibitor MG132) on NKG2D ligands and the susceptibility of A549 cells to NK lysis. The possible regulatory mechanisms underlying the DNA damage response pathway were also examined.

\section{Materials and methods}

Cell culture and reagents. The A549 (catalog no. 3111C0001CCC00002), NCI-H520 (catalog no. 3111C0001CCC000197) and NCI-H157 (catalog no. 3111C0001CCC000113) lung cancer cell lines were purchased from Peking Union Culture Collection (Beijing, China). The PLA801D lung cancer cell line (catalog no. CBP61005) was purchased from Nanjing Cobioer Biotechnology Corporation (Nanjing, China). The cells were maintained in RPMI-1640 (Gibco; Thermo Fisher Scientific, Inc., Waltham, MA, USA), supplemented with $100 \mathrm{IU} / \mathrm{ml}$ penicillin, $100 \mu \mathrm{g} / \mathrm{ml}$ streptomycin and $10 \%$ fetal bovine serum (Gibco; Thermo Fisher Scientific, Inc.), and incubated at $37^{\circ} \mathrm{C}$ with $5 \% \mathrm{CO}_{2}$. The NK92 cell line was purchased from the Peking Union Culture Collection and cultured at $37^{\circ} \mathrm{C}$ with $5 \% \mathrm{CO}_{2}$ in $\alpha$-MEM medium (Gibco; Thermo Fisher Scientific, Inc.) supplemented with $12.5 \%$ fetal bovine serum and $12.5 \%$ horse serum (Hyclone; GE Healthcare, Inc., Logan, UT, USA). MG132, KU-55933, caffeine and wortmannin were purchased from Sigma-Aldrich (Merck KGaA, Darmstadt, Germany). Mouse anti-human MICA (catalog no. sc-23870), MICB (catalog no. sc-80527), ULBP1 (catalog no. sc-53131), ULBP2 (catalog no. sc-53135), ULBP3 (catalog no. sc-53132) and ULBP4 (catalog no. sc-53133) monoclonal antibodies (mAbs) were purchased from Santa Cruz Biotechnology, Inc. (Dallas, TX, USA). FITC-labeled goat anti-mouse IgG (catalog no. ab6785) was purchased from Abcam (Cambridge, MA, USA). Anti-Chk2 (catalog no. 2662) and -p-Chk2 (catalog no. 2661) mAbs were purchased from Cell Signaling Technology, Inc. (Danvers, MA, USA).

Treatment of the cells with the inhibitors. A549 cells were treated with KU-55933 $(10 \mu \mathrm{M})$ for $9 \mathrm{~h}$ or wortmannin $(6 \mu \mathrm{M})$ or caffeine $(10 \mathrm{mM})$ for $10 \mathrm{~h}$, or MG132 for $8 \mathrm{~h}$, or with KU-55933 for $9 \mathrm{~h}$ or wortmannin or caffeine for $10 \mathrm{~h}$, with the addition of MG132 during the final $8 \mathrm{~h}$ at $37^{\circ} \mathrm{C}$. Then cells were harvested at $300 \mathrm{xg}$ at room temperature for $5 \mathrm{~min}$.

Flow cytometry. The cells were harvested, washed three times with PBS, and adjusted to a concentration of $5 \times 10^{5}$ cells $/ \mathrm{ml}$ with PBS. The cell suspensions $(200 \mu \mathrm{l})$ were added to labeled tubes and stained with mouse anti-human MICA, MICB, ULBP1, ULBP2, ULBP3 and ULBP4 antibodies (1:200) or an isotype antibody for $30 \mathrm{~min}$ at $4^{\circ} \mathrm{C}$, followed by incubation with goat anti-mouse $\operatorname{IgG}$ in the dark. Following incubation at $4^{\circ} \mathrm{C}$ for $30 \mathrm{~min}$, the cells were washed three times in PBS. The samples were examined using FACSCalibur flow cytometry (BD Biosciences, Franklin Lakes, NJ, USA). Data were analyzed with FlowJo version 7.6 software (FlowJo LLC, Ashland, OR, USA).

Promoter analysis. The genomic DNA of the A549 cells was isolated and the full-length MICB promoter was amplified using polymerase chain reaction (PCR). The promoter fragments were cloned into the pGL3 luciferase reporter plasmid. The A549 cells were transfected with $0.79 \mu \mathrm{g}$ MICB promoter plasmid and $0.01 \mu \mathrm{g} \mathrm{SV} 40$-Renilla plasmid using Lipofectamine ${ }^{\circledR} 2000$ and Plus reagents (Invitrogen; Thermo Fisher Scientific, Inc.) according to the manufacturer's protocol. The cells $\left(5 \times 10^{6}\right)$ were cultured at $37^{\circ} \mathrm{C}$ for $24 \mathrm{~h}$, following which they were treated with $10 \mu \mathrm{M} \mathrm{MG132}$ at $37^{\circ} \mathrm{C}$ for $8 \mathrm{~h}$ and then lysed (Promega Corporation, Madison, WI, USA). Luciferase and Renilla activity were measured as previously described (22).

Reverse transcription-quantitative PCR (RT-qPCR) analysis. RNA was isolated using TRIzol $^{\circledR}$ reagent (Invitrogen; Thermo Fisher Scientific, Inc.) according to the manufacturer's protocol (23). RT of $2 \mu \mathrm{g}(20 \mu \mathrm{l}) \mathrm{RNA}$ into cDNA was performed using PrimeScript ${ }^{\mathrm{TM}}$ Reverse Transcriptase (Takara Biotechnology Co., Ltd., Dalian, China). MICA, MICB, ULBP1 and ULBP2 PCR (cDNA $50 \mathrm{ng}, 0.5 \mu \mathrm{l}$ ) was performed with buffer TB Green Premix Ex Taq II (Takara Biotechnology Co., Ltd.) under the following cycling conditions: $94^{\circ} \mathrm{C}$ for $40 \mathrm{sec}, 61^{\circ} \mathrm{C}$ for $40 \mathrm{sec}, 72^{\circ} \mathrm{C}$ for $50 \mathrm{sec}$, and extension at $72^{\circ} \mathrm{C}$ for $10 \mathrm{~min}$ for 40 cycles. The quantification of the NKG2D ligands and $\beta$-actin was performed using specific primers and the sequences were as follows: MICA, upstream, 5'-CGGGAT CCTTTCTCACTGAGGTACAT-3' and downstream 5'-CGG AATTCTGTCACGGTAATGTTGCC-3'; MICB, upstream 5'-CGGGATCCCACAGTCTTCGTTACAAC-3' and downstream 5'-CGGAATTCCTATGTCACGGTGATGTTGC-3'; ULBP1, upstream 5'-CGGGATCCACACACTGTCTTTGCT AT-3' and downstream 5'-CGGAATTCTCACAGCATTTGT TCCCAGTA-3'; ULBP2, upstream 5'-CGGGATCCGACC 
CTCACTCTCTTTGC-3' and downstream 5'-CGGAATTCG AGGAGGAAGATCTGCC-3'; and $\beta$-actin, upstream 5'-ATC ATGTTTGAGACCTTCAACA-3' and downstream 5'-CAT CTCTTGCTCGAAGTC-3'. The percentage change was calculated using the following formula: $2^{-\Delta \Delta \mathrm{Cq}}(24)$.

Cytotoxicity assays. The cytotoxicity of the NK cells was measured using a standard ${ }^{51} \mathrm{Cr}$-release assay (25). Briefly, the target tumor cells were incubated for $1 \mathrm{~h}$ with $150 \mu \mathrm{Ci}$ ${ }^{51} \mathrm{Cr}$ (PerkinElmer, Inc., Waltham, MA, USA) at $37^{\circ} \mathrm{C}$ in $5 \% \mathrm{CO}_{2}$. The cells were then washed three times with media and incubated for an additional $30 \mathrm{~min}$. In order to detect the differential lysis effect of different effector to target cell ratios, labeled target cells $\left(1 \times 10^{4}\right.$ cells/well $)$ were incubated with effector cells in 96-well plates in 10\% FCS-RPMI-1640 at a total volume of $200 \mu \mathrm{l}$. The plates were centrifuged at $300 \mathrm{x} \mathrm{g}$ at $37^{\circ} \mathrm{C}$ for $5 \mathrm{~min}$ following incubation for $4 \mathrm{~h}$. Aliquots $(100 \mu \mathrm{l})$ of the supernatants from each well were transferred to a new plate containing $100 \mu 1 /$ well of Optiphase Supermix scintillation fluid. The NK cells were pre-incubated at $37^{\circ} \mathrm{C}$ for $1 \mathrm{~h}$ with NKG2D antibodies (dilution 1:500) for antibody blocking experiments. Radioactivity was measured using a gamma counter. The percentage of cytotoxicity was calculated according to the following formula: 100x (experimental release-spontaneous release)/(maximum release-spontaneous release). Maximum release was determined by the addition of $100 \mu 1$ 10\% Triton X-100 and spontaneous release was determined by incubating the targets with $100 \mu \mathrm{l}$ complete media.

Comet assay. The alkaline comet method of Singh et al (26) was followed with minor differences, and the application steps described. The cells were harvested following treatment with $10 \mu \mathrm{M}$ MG132 for $8 \mathrm{~h}$. The slides were pre-coated with $1 \%$ regular agarose. A low-melting-point agarose $(0.65 \%)$ suspension was added to the cell suspension at a ratio of $4: 1$ and the suspension was immediately transferred onto the slides. The cells on the slides were lysed with ice-cold high-salt lysis buffer $(2.5 \mathrm{M} \mathrm{NaCl}, 100 \mathrm{mM}$ EDTA, $10 \mathrm{mM}$ Tris $\mathrm{pH} 10,1 \%$ Triton X-100 and 10\% DMSO) in the dark at $4^{\circ} \mathrm{C}$ for $1 \mathrm{~h}$ for disintegration of the cell and nuclear membranes. Following the lysis phase, the slides were placed in an electrophoresis tank with electrophoresis buffer ( $\mathrm{pH} 10.0$ ) and incubated in the dark and $4^{\circ} \mathrm{C}$ for $30 \mathrm{~min}$. Electrophoresis was then performed at $25 \mathrm{~V}$ for $20 \mathrm{~min}$. Subsequently, the slides were washed twice for $5 \mathrm{~min}$ with neutralization buffer and allowed to air-dry until analysis. All samples were evaluated within $24 \mathrm{~h}$ and were not subjected to fixation. The samples were stained with $25 \mu \mathrm{g} / \mathrm{ml}$ propidium iodide and then visualized under a fluorescence microscope (Leica Microsystems GmbH, Wetzlar, Germany) and analyzed with CASPLab version 1.2.2 software (University of Wroclaw, Wroclaw, Poland) (27).

Western blotting. Tumor cells were collected, washed three times with PBS and then lysed with RIPA buffer (Beyotime Institute of Biotechnology, Shanghai, China) for $30 \mathrm{~min}$ at $4^{\circ} \mathrm{C}$. Subsequently, the suspension was centrifuged at $16,000 \mathrm{x} \mathrm{g}$ for $15 \mathrm{~min}$ at $4^{\circ} \mathrm{C}$. The concentration of proteins was detected using a BCA assay kit (Sigma-Aldrich; Merck KGaA). Equal quantities $(30 \mu \mathrm{g})$ of protein were separated using SDS-PAGE (10\% gels) and transferred onto polyvinylidene fluoride membranes under $100 \mathrm{~V}$ for $1 \mathrm{~h}$. The membranes were blocked using 5\% non-fat dry milk for $1 \mathrm{~h}$ at room temperature. Subsequently, the membranes were blotted with an appropriate primary antibody (Anti-Chk2, dilution 1:1,000, catalog no. 2662 and p-Chk2, dilution 1:1,000, catalog no. 2661, both Cell Signaling Technology, Inc.) overnight at $4^{\circ} \mathrm{C}$. The membranes were washed with TBST (TBS, pH 7.5, containing $0.05 \%$ Tween-20) and were then incubated with horseradish peroxidase-conjugated secondary antibodies $(1: 1,500)$ at room temperature for $1 \mathrm{~h}$. The blots were visualized using ECL reagents (Beyotime Institute of Biotechnology).

Statistical analysis. All results presented are representative of a minimum of three experiments. Statistical comparisons between groups were made using Student's t-test or one-way analysis of variance followed by NewmanKeul's post hoc test. $\mathrm{P}<0.05$ was considered to indicate a statistically significant difference. Statistical analysis was calculated using GraphPad, version 5.0 (GraphPad Software, Inc., La Jolla, CA, USA).

\section{Results}

A restricted set of NKG2D ligands are expressed in the A549 cell line. The expression levels of cell surface NKG2D ligands were measured in the lung cancer cell lines using flow cytometry (Fig. 1). The results demonstrated that the expression levels of NKG2D ligands in PLA801D, NCI-H520, NCI-H157 and A549 cells were different (Table I). In the A549 cells, MICA/B and ULBP1 were weakly expressed, ULBP2 showed typical expression, and neither ULBP3 nor ULBP4 were expressed (Table I).

NKG2D ligand expression is selectively induced by MG132. To investigate whether the cancer treatment agent MG132 affects NKG2D ligands, the expression levels of NKG2D ligands were measured following treatment with MG132 (22). Following treatment with MG132 for $8 \mathrm{~h}$, the transcription levels of MICB and ULBP1 were upregulated by 10.62- and 11.09-fold, respectively (Fig. 2A), and the surface expression levels of MICB and ULBP1 were increased by 68.18 and $23.65 \%$, respectively (Fig. 2B and C). Among the ligands, the expression of MICB exhibited the most marked change. Following treatment with MG132, the mRNA levels of MICB increased linearly between 0 and $8 \mathrm{~h}$ and slowly increased after $8 \mathrm{~h}$ (Fig. 3A). In addition, following stimulation with MG132, MICB was not detectable between 2 and 4 h, however, the expression of MICB rapidly increased between 4 and $8 \mathrm{~h}$ and then slowly increased after $8 \mathrm{~h}$ (Fig. 3B), which indicated that the MG132-induced expression of MICB is time-dependent.

MICB promoter is activated by a proteasome inhibitor. As the proteasome inhibitor induced the transcription of MICB, whether this change is initiated at the MICB promoter was subsequently investigated. Promoter fragments of MICB, from the MICB translation start site ATG to 480-bp upstream, were cloned into the luciferase reporter vector pGL3 (28). The A549 cells transfected with the pGL3-luciferase vector and incubated with MG132 for $8 \mathrm{~h}$ prior to harvesting exhibited increased luciferase activity and a 1.77 -fold increase in promoter activity, as corrected for transfection efficiency using the co-transfected pRL-SV40 (Renilla luciferase) plasmid 
Table I. Mean fluorescence intensity of NK group 2, member D ligands on non-small cell lung cancer cells.

\begin{tabular}{lcccccc}
\hline Cells & MICA & MICB & ULBP1 & ULBP2 & ULBP3 & ULBP4 \\
\hline A549 & 1.34 & 1.27 & 1.22 & 1.80 & 0.95 & 1.03 \\
PLA801D & 1.77 & 1.49 & 2.11 & 1.14 & 1.47 & 1.04 \\
NCI-H157 & 1.03 & 3.83 & 1.10 & 1.92 & 1.10 & 1.04 \\
NCI-H520 & 1.76 & 1.14 & 2.04 & 2.79 & 2.99 & 1.06 \\
\hline
\end{tabular}

MIC, MHC class I polypeptiderelated sequence A; ULBP, UL16 binding protein.
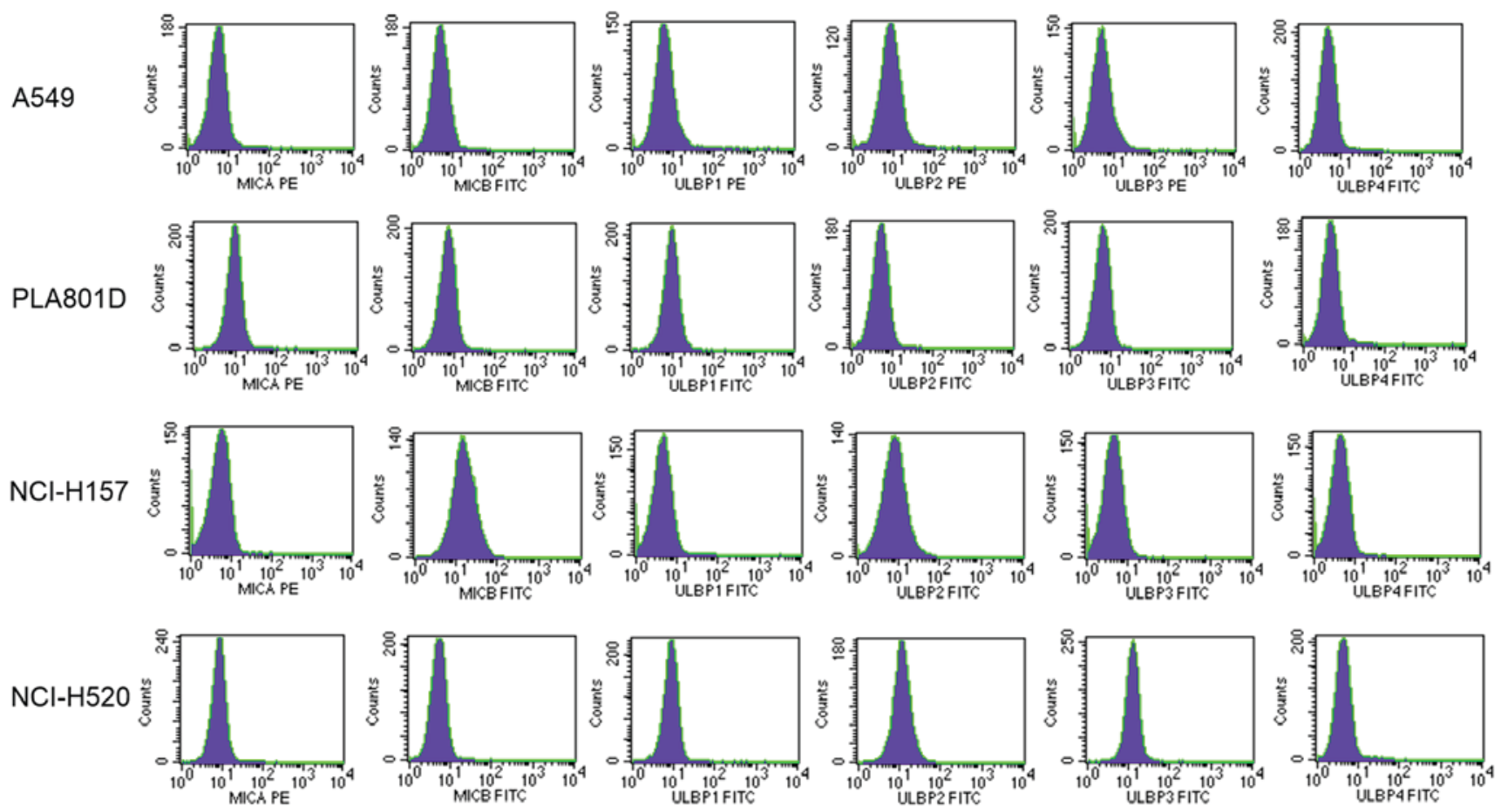

Figure 1. Expression of NKG2D ligands on non-small cell lung cancer cells. The values of mean fluorescence intensity of the NKG2D ligands described in Table I. NKG2D, NK group 2, member D.

(Fig. 3C). These results indicate that MG132 increases the transcription of MICB and increases the activity of the MICB promoter.

NKG2D-mediated tumor cell lysis is enhanced by the upregulation of MICB. To determine the effect of increased levels of NKG2D ligands in tumor cells, the cytotoxicity of NK cells against A549 cells pretreated with MG132 was measured. As shown in Fig. 4, MG132 significantly increased the susceptibility of the A549 cell line to cytolysis by NK cells. When the NKG2D-NKG2D ligand interactions were blocked with anti-NKG2D antibody, the lysis of A549 cells treated with MG132 was markedly reduced (Fig. 4A). The increased lysis of the MG132-treated cells was partially blocked by the MICB antibody (Fig. 4B). These results indicate that the interaction between NKG2D and its ligands is important in the NK-mediated lysis of the A549 cell line, and that the increased susceptibility of MG132-treated cancer cells to the cytotoxicity of NK cells may be mediated by upregulation of the NKG2D ligand MICB.
MG132 induces DNA damage in A549 cells. Previous studies have demonstrated that genotoxic agents that activate the DNA damage response pathway are responsible for the upregulation of NKG2D ligand expression in numerous tumor cell lines $(13,22,23)$. Several of the chemotherapeutic drugs used clinically have the ability to induce the activation of ATM. Therefore, it was hypothesized that the MG132-induced upregulation of MICB in A549 cells may be dependent on activation of the DNA damage response pathway. Following MG132 treatment, the results produced a 'comet tail' in the comet assay, which indicates DNA strand breakage (Fig. 5A-C).

Chk2 is activated by MG132 in A549 cells. Numerous types of cancer cell, including A549 cells, exhibit defective DNA repair mechanisms. Chk2 autophosphorylation at Thr68 is a key early signaling event in the DNA damage response cascade $(22,29)$. Therefore, whether Chk 2 was functionally activated in MG132-treated A549 cells was investigated in the present study. The A549 cells were treated with $10 \mu \mathrm{M}$ MG132 for $8 \mathrm{~h}$ and lysed, following which the phosphorylation of Chk2 at Thr68 

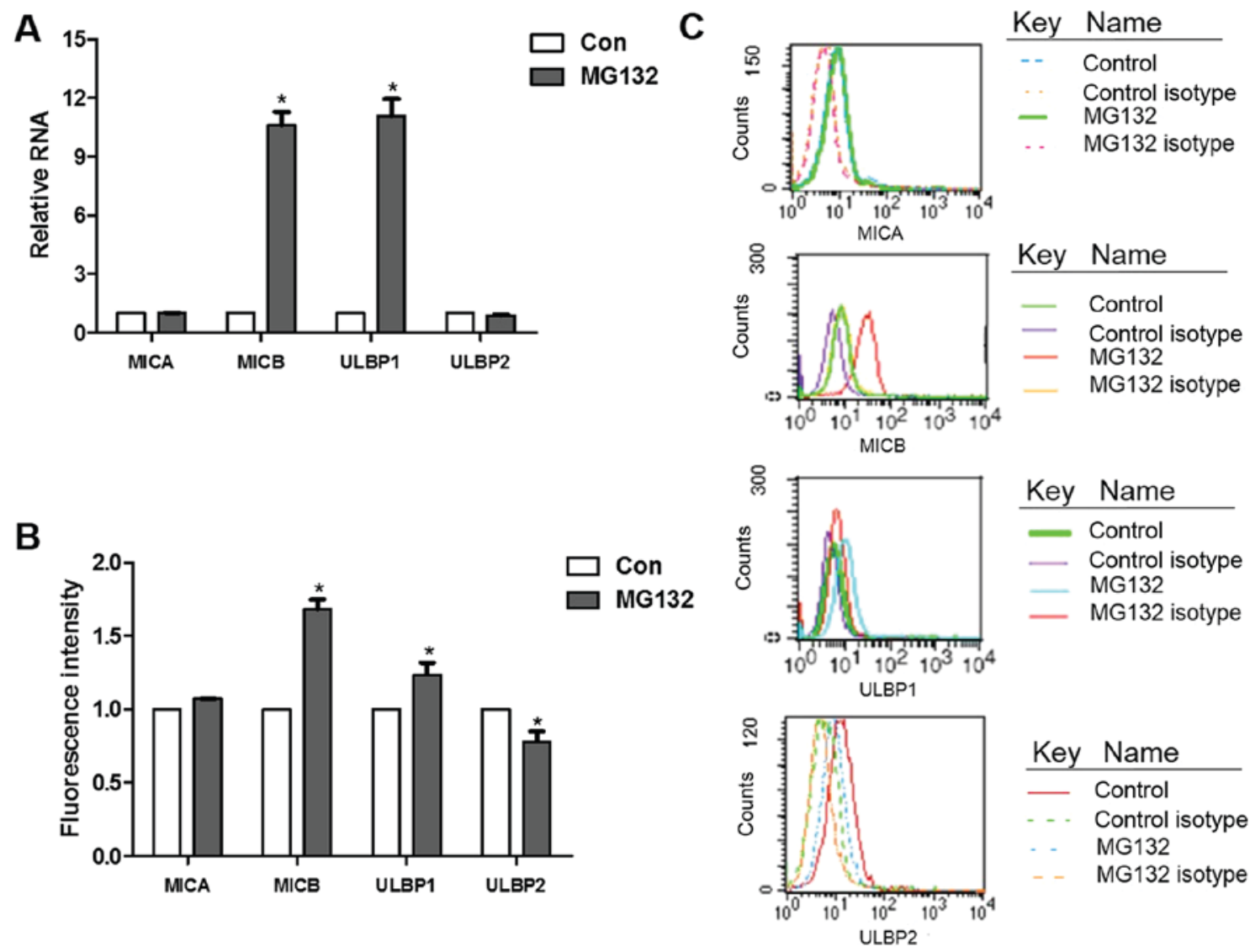

Figure 2. MG132 selectively induces the expression of NKG2D ligands. A549 cells were incubated with $10 \mu \mathrm{M}$ MG132 for $8 \mathrm{~h}$, and then (A) the mRNA expression of NKG2D ligands was detected using reverse transcription-quantitative polymerase chain reaction analysis and the (B) cell surface expression of NKG2D ligands was assessed via (C) flow cytometry. Data are representative of three independent experiments. Comparisons of two groups was performed with Student's t-test. "P<0.05. NKG2D, NK group 2, member D; Con, control.

A

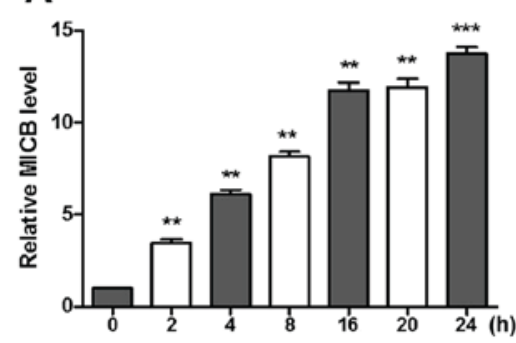

B

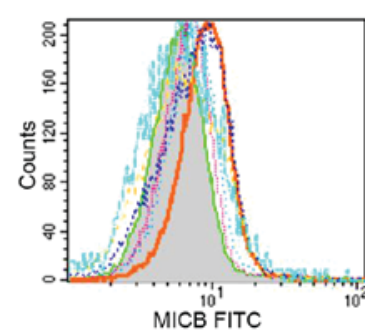

C

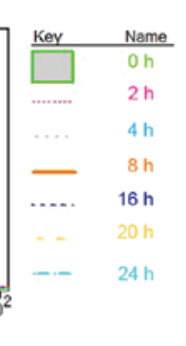

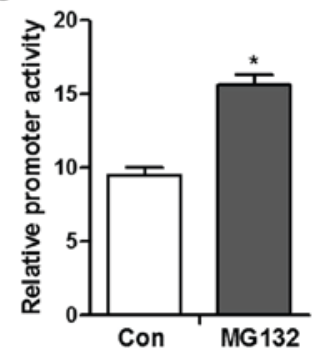

Figure 3. MG132 induces the expression of MICB and increases MICB promoter activity in A549 cells. (A) A549 cells were treated with DMSO solvent or MG132 for the durations indicated, followed by cell lysis and analysis of the mRNA expression of MICB. (B) Anti-MICB monoclonal antibody staining of A549 cells treated with MG132 for the durations indicated on a logarithmic scale. Expression of MICB at $8 \mathrm{~h}$ is at the top. (C) A549 cells were transfected with the indicated pGL3-luciferase plasmids. The co-transfected pRL-TK plasmid was used as a control for transfection efficiency. MICB promoter pGL3-luciferase activity was assessed. At $32 \mathrm{~h}$ post-transfection, the cells were cultured with DMSO or MG132 for an additional $8 \mathrm{~h}$ followed by lysis. The histogram shows the relative increase in activity. Comparison of two groups was performed using Student's t-test. Multiple comparisons were performed with one-way analysis of variance. ${ }^{*} \mathrm{P}<0.05,{ }^{* *} \mathrm{P}<0.01$ and ${ }^{* * *} \mathrm{P}<0.001$. MIC, MHC class I polypeptiderelated sequence; Con, control.

was measured using western blotting. The results demonstrated that the phosphorylation of Chk2 at Thr68 was induced by $10 \mu \mathrm{M}$ MG132 (Fig. 6). Although other aspects of the DNA damage response pathway have not been excluded, these results indicate that the autophosphorylation of $\mathrm{Chk} 2$ is involved in the increased expression of MICB induced by MG132.

MG132-induced expression of MICB is eliminated following treatment with KU-55933 (ATM kinase inhibitor), wortmannin [phosphoinositide 3 (PI3) kinase inhibitor] and caffeine (ATM/R inhibitor). Gasser et al (30) demonstrated that the expression of NKG2D ligands is induced by ATM/ATM-Rad3-related (ATR) signaling in the DNA damage response pathway and that induction is prevented by ATM/ATR inhibitors, including caffeine. Therefore, whether the ATM/ATR inhibitors KU-55933, wortmannin and caffeine can prevent drug-induced MICB transcription was investigated in the present study. Treatment with KU-55933, wortmannin 
A

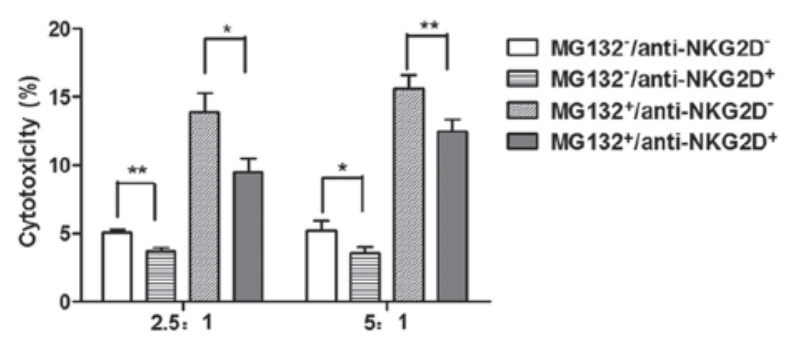

B

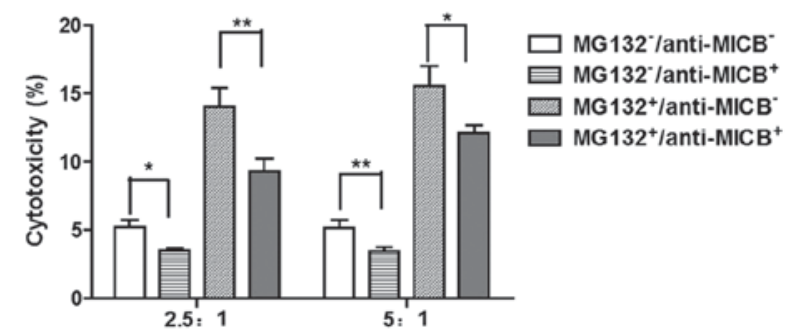

Figure 4. MICB enhances NK cell lysis of MG132-treated A549 cells. The cytotoxicity of NK cells against the A549 cell line was measured at different effector/target cell ratios with a $4-\mathrm{h}{ }^{51} \mathrm{Cr}$-release assay. A549 cells were stimulated with $10 \mu \mathrm{M}$ MG132 for $8 \mathrm{~h}$, and then washed and used as the target cells. For the NKG2D antibody inhibition control experiments, tumor cells that had been stimulated with MG132 were washed completely prior to the NK lysis assay. (A) Increased lysis of the MG132-treated cells was partially inhibited by the NKG2D antibody. Tumor cells were stimulated with MG132, incubated with the anti-MICB mAb for $1 \mathrm{~h}$, and then washed entirely prior to the NK lysis assay. (B) Increased lysis of the MG132-treated cells was partially inhibited by the MICB mAb. Multiple comparisons were performed with one-way analysis of variance. ${ }^{*} \mathrm{P}<0.05$ and ${ }^{* *} \mathrm{P}<0.01$. MIC, MHC class I polypeptiderelated sequence; NK, natural killer; NKG2D, NK group 2, member D; mAb, monoclonal antibody.

A

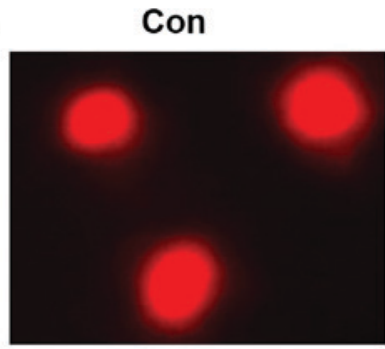

MG132

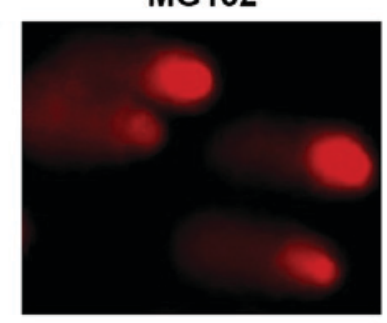

B

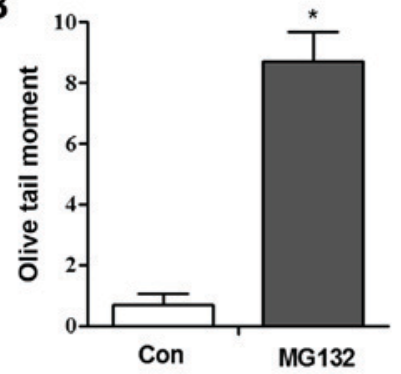

C

\begin{tabular}{ccc}
\hline & \multicolumn{2}{c}{ Olive tail moment } \\
\hline & Con & MG132 \\
MG132 & $0.7019 \pm 0.3701$ & $8.7299 \pm 0.9432$ \\
\hline
\end{tabular}

Figure 5. MG132 induces DNA damage in A549 cells. (A) Representative comet assay demonstrating the formation of DNA strand breaks, as shown by the formation of a 'comet tail' (magnification, x200). (B) Fraction of cells containing a comet tail. Data are presented as the mean \pm standard deviation. (C) Olive tail moment following treatment with MG132. Comparison of two groups was performed using Student's t-test. "P<0.05. Con, control.

and caffeine inhibited the MG132-induced upregulation of $M I C B$ (Fig. 7A). Consistent with the RT-qPCR results, the flow cytometry revealed a similar trend (Fig. 7B). These results indicate that the ATM/ATR signaling pathway is a possible mechanism by which MG132 induces the expression of MICB.

\section{Discussion}

In experimental animals and patients with cancer, the expression of tumor NKG2D ligands is associated with tumor eradication and survival rate (22). The expression levels of NKG2D ligands are increased in tumor cells compared with those in the surrounding normal tissue (21), which can be induced further by cancer treatment agents $(30,31)$. Therefore, effective cancer treatments may directly damage tumor cells and induce the expression of NKG2D ligands, causing NK cell attack. In the present study, the expression levels of NKG2D ligands in A549 cells and other lung cancer cell lines, including PLA801D, NCI-H520 and NCI-H157, were detected. The results demonstrated that different lung cancer cell lines express different NKG2D ligands and have different levels of sensitivity to NKG2D-mediated cell death by NK cells. The regulation

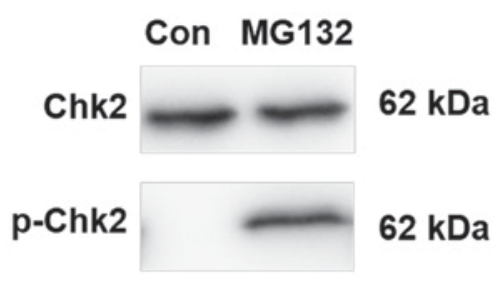

$\beta$-actin

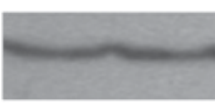

$42 \mathrm{kDa}$

Figure 6. Chk2 is phosphorylated at Thr68 in A549 cells treated with MG132. A549 cells were treated with MG132 for $8 \mathrm{~h}$ followed by lysis and then western blotting. Phospho-Chk2 at Thr68 was detected. The same membrane was stripped and reprobed with $\beta$-actin antibody as the control. Chk2, checkpoint kinase 2; p-Chk2, phosphorylated Chk2; Con, control.

of NKG2D ligands in human A549 cells, a tumor system that remains to be fully elucidated, was investigated. The regulation of NKG2D ligands by the proteasome inhibitor MG132 and the susceptibility of A549 cells to NK lysis were also investigated. The possible regulatory mechanisms involved were examined, focusing on the role of the DNA damage response pathway. 
A

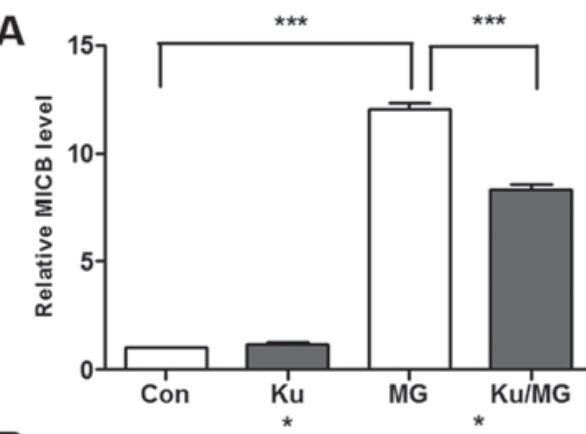

B

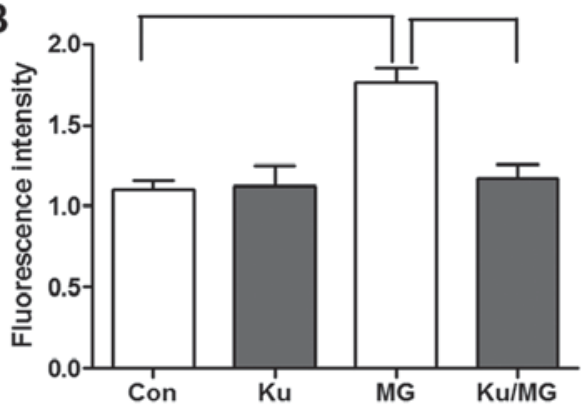

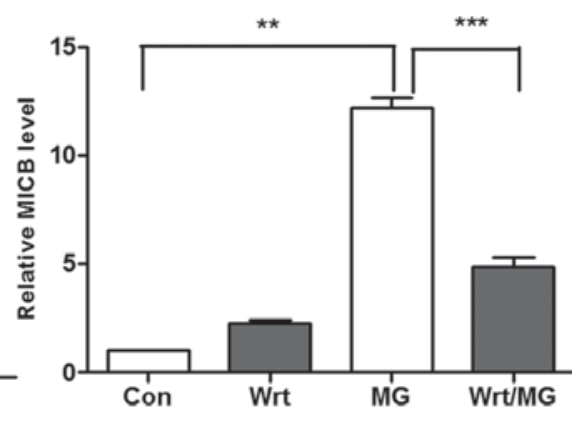
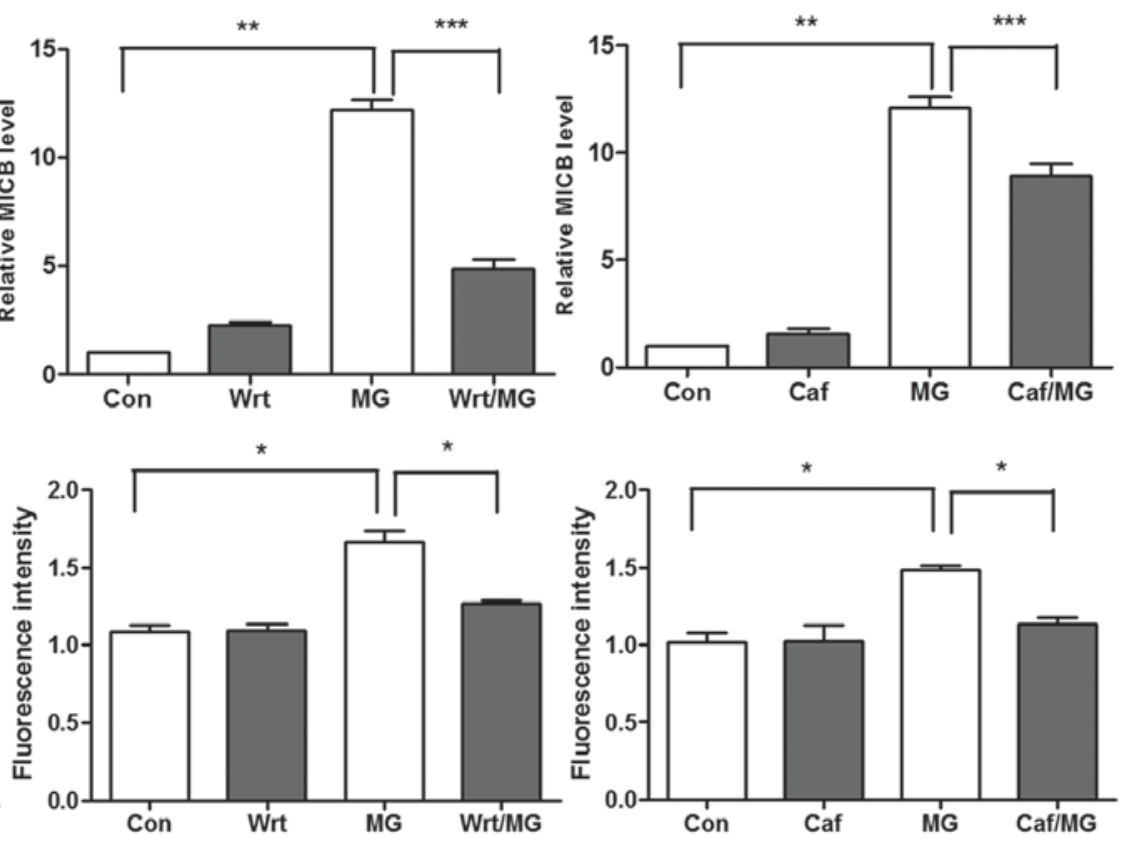

Figure 7. ATM kinase inhibitor (KU-55933), phosphoinositide 3 kinase inhibitor (wortmannin) and ATM/ATM-Rad3-related inhibitor (caffeine) prevent MG132-induced upregulation of MICB. A549 cells were treated with KU-55933 (10 $\mu \mathrm{M})$ for $9 \mathrm{~h}$ or wortmannin $(6 \mu \mathrm{M})$ or caffeine (10 mM) for $10 \mathrm{~h}$, or MG132 for $8 \mathrm{~h}$, or with KU-55933 for $9 \mathrm{~h}$ or wortmannin or caffeine for $10 \mathrm{~h}$, with the addition of MG132 during the final $8 \mathrm{~h}$. The expression of MICB was measured and was normalized to that of control DMSO-treated cells (Con). (A) RNA levels and (B) flow cytometry. Multiple comparisons were performed with one-way analysis of variance. ${ }^{*} \mathrm{P}<0.05,{ }^{* *} \mathrm{P}<0.01$ and ${ }^{* * * *} \mathrm{P}<0.001$. MIC, MHC class I polypeptiderelated sequence; Ku, KU-55933; Wrt, wortmannin; Caf, caffeine; MG, MG132; Con, control.

Proteasome inhibitors have attracted interest as a novel type of antitumor drug. MG132 is an aldosterone inhibitor that reversibly inhibits proteasome chymotrypsin-like activity, inhibiting its degradation of protein substrates, inhibiting tumor cell growth and inducing apoptosis. MG132 is a potential therapeutic and preventive agent for cancer cachexia $(32,33)$. Butler et al $(22)$ reported that proteasome inhibitors that regulate NKG2D ligands are multifaceted, multilevel and can selectively upregulate expression of the ULBP1 promoter, and mRNA and cell surface proteins in head and neck squamous cell carcinoma. As the role of MG132 is complicated, the regulation of MICB may also be multilinked and multilevel. Therefore, the effects of MG132 on the promoter activity, mRNA expression and cell surface expression of MICB were investigated in the present study. Whether MG132 can cause DNA damage and activate key molecules in the DNA damage response pathway were also examined. The results demonstrated that MG132 upregulated the activity of the MICB promoter, mRNA expression and cell surface protein expression. Additionally, MG132 induced DNA single-strand breaks and activated Chk2 molecules. KU-55933 (ATM kinase inhibitor), wortmannin (PI3 kinase inhibitor) and caffeine (ATM/R inhibitor) inhibited the expression of MICB that was induced by MG132. These results indicate that DNA damage induced by MG132 activates protein kinase molecules, including ATM and Chk2, and those of other DNA damage pathways, which may affect the expression of MICB on the cell surface.

Based on analysis with Gene Runner software and experimental data (28), it was hypothesized that the core region of the MICB promoter includes elements for transcription regulation, including TATA-like elements and HSE. These elements may regulate the transcription of $\mathrm{MICB}$, and this requires further investigation. Winter et al (34) reported that, following DNA damage, ATM molecules can phosphorylate nuclear E3 ubiquitin ligase Siah-1 and inhibit Siah-1-mediated homeodomain-interacting protein 2 (HIPK2) ubiquitination and degradation. HIPK 2 activates phosphorylated p53 and promotes apoptosis. Therefore, the role of MG132 is associated not only with the DNA damage response but also with the ubiquitination and degradation of signaling molecules. However, the detailed mechanism requires further investigation.

In conclusion, the present study demonstrates that MG132 selectively upregulates the surface expression of MICB in A549 cells, and increases the NKG2D-mediated cytotoxicity of NK cells. The regulatory effect of MG132 is associated with the activation of Chk2, an event associated with DNA damage. The combination of MG132 with NK cell immunotherapy may have a synergistic effect that improves the therapeutic effect of lung cancer treatment.

\section{Acknowledgements}

Not applicable.

\section{Funding}

This study was supported by the Natural Science Foundation of China (grant no. 81503391), the China Youth Foundation (grant no. 31500137) and the China Postdoctoral Science Foundation (grant no. 2015M582847).

\section{Availability of data and materials}

All data generated or analyzed during the present study are included in this published article. 


\section{Authors' contributions}

ZNC, FL and ZLW conceived and designed the experiments. DL, XWD and BY performed the experiments and drafted the manuscript. ML and JHY were involved in the data analysis. HL and THX assisted with the experiments. All authors reviewed and approved the final manuscript.

\section{Ethics approval and consent to participate}

Not applicable.

\section{Patient consent for publication}

Not applicable.

\section{Competing interests}

The authors declare that they have no competing interests.

\section{References}

1. Bellora F, Castriconi R, Dondero A, Carrega P, Mantovani A Ferlazzo G, Moretta A and Bottino C: Human NK cells and NK receptors. Immunol Lett 161: 168-173, 2014.

2. Gasser S and Raulet DH: Activation and self-tolerance of natural killer cells. Immunol Rev 214: 130-142, 2006.

3. Bae DS, Hwang YK and Lee JK: Importance of NKG2D-NKG2D ligands interaction for cytolytic activity of natural killer cell. Cell Immunol 276: 122-127, 2012

4. Kruse PH, Matta J, Ugolini S and Vivier E: Natural cytotoxicity receptors and their ligands. Immunol Cell Biol 92: 221-229, 2014

5. Yin X, Lu X, Zhang X, Min Z, Xiao R, Mao Z and Zhang Q: Role of NKG2D in cytokine-induced killer cells against lung cancer. Oncol Lett 13: 3139-3143, 2017.

6. Mayes K, Elsayed Z, Alhazmi A, Waters M, Alkhatib SG, Roberts M, Song C, Peterson K, Chan V, Ailaney N, et al: BPTF inhibits NK cell activity and the abundance of natural cytotoxicity receptor co-ligand. Oncotarget 8: 64344-64357, 2017.

7. Borchers MT, Harris NL, Wesselkamper SC, Vitucci M and Cosman D: NKG2D ligands are expressed on stressed human airway epithelial cells. Am J Physiol Lung Cell Mol Physiol 291: L222-L231, 2006.

8. Burgess SJ, Maasho K, Masilamani M, Narayanan S, Borrego F and Coligan JE: The NKG2D receptor: Immunobiology and clinical implications. Immunol Res 40: 18-34, 2008.

9. Coudert JD and Held W: The role of the NKG2D receptor for tumor immunity. Semin Cancer Biol 16: 333-343, 2006.

10. Champsaur M and Lanier LL: Effect of NKG2D ligand expression on host immune responses. Immunol Rev 235: 267-285, 2010.

11. Stern-Ginossar N, Elefant N, Zimmermann A, Wolf DG, Saleh N, Biton M, Horwitz E, Prokocimer Z, Prichardi M, Hahn G, et al: Host immune system gene targeting by a viral miRNA. Science 317: 376-381, 2007.

12. Bauer S, Groh V, Wu J, Steinle A, Phillips JH, Lanier LL and Spies T: Activation of NK cells and T cells by NKG2D, a receptor for stress-inducible MICA. Science 285: 727-729, 1999.

13. Fionda C, Soriani A, Malgarini G, Iannitto ML, Santoni A and Cippitelli M: Heat shock protein-90 inhibitors increase MHC class I-related chain A and B ligand expression on multiple myeloma cells and their ability to trigger NK cell degranulation. J Immunol 183: 4385-4394, 2009.

14. Haque M, Ueda K, Nakano K, Hirata Y, Parravicini C, Corbellino $\mathrm{M}$ and Yamanishi $\mathrm{K}$ : Major histocompatibility complex class I molecules are down-regulated at the cell surface by the K5 protein encoded by Kaposi's sarcoma-associated herpesvirus/human herpesvirus-8. J Gen Virol 82: 1175-1180, 2001.
15. Friese MA, Wischhusen J, Wick W, Weiler M, Eisele G, Steinle A and Weller M: RNA interference targeting transforming growth factor-beta enhances NKG2D-mediated antiglioma immune response, inhibits glioma cell migration and invasiveness, and abrogates tumorigenicity in vivo. Cancer Res 64: 7596-7603, 2004.

16. Nachmani D, Stern-Ginossar N, Sarid R and Mandelboim O: Diverse herpesvirus microRNAs target the stress-induced immune ligand MICB to escape recognition by natural killer cells. Cell Host Microbe 5: 376-385, 2009.

17. Lanier LL: NKG2D receptor and its ligands in host defense. Cancer Immunol Res 3: 575-582, 2015.

18. Stern-Ginossar N, Gur C, Biton M, Horwitz E, Elboim M, Stanietsky N, Mandelboim M and Mandelboim O: Human microRNAs regulate stress-induced immune responses mediated by the receptor NKG2D. Nat Immunol 9: 1065-1073, 2008.

19. Morisaki T, Onishi $\mathrm{H}$ and Katano M: Cancer immunotherapy using NKG2D and DNAM-1 systems. Anticancer Res 32: 2241-2247, 2012.

20. Tallerico R, Todaro M, Di FS, Maccalli C, Garofalo C, Sottile R, Palmieri C, Tirinato L, Pangigadde PN and La Rocca R: Human NK cells selective targeting of colon cancer-initiating cells: A role for natural cytotoxicity receptors and MHC class I molecules. J Immunol 190: 2381-2390, 2013.

21. Wu J, Song Y, Bakker AB, Bauer S, Spies T, Lanier LL and Phillips JH: An activating immunoreceptor complex formed by NKG2D and DAP10. Science 285: 730-732, 1999.

22. Butler JE, Moore MB, Presnell SR, Chan HW, Chalupny NJ and Lutz CT: Proteasome regulation of ULBP1 transcription. J Immunol 182: 6600-6609, 2009.

23. Zhang C, Wang Y, Zhou Z, Zhang J and Tian Z: Sodium butyrate upregulates expression of NKG2D ligand MICA/B in HeLa and HepG2 cell lines and increases their susceptibility to NK lysis. Cancer Immunol Immunother 58: 1275-1285, 2009.

24. Livak KJ and Schmittgen TD: Analysis of relative gene expression data using real-time quantitative PCR and the 2- $\Delta \Delta \mathrm{CT}$ method. Methods 25: 402-408, 2001.

25. Maniar A, Zhang X, Lin W, Gastman BR, Pauza CD, Strome SE and Chapoval AI: Human gammadelta T lymphocytes induce robust NK cell-mediated antitumor cytotoxicity through CD137 engagement. Blood 116: 1726-1733, 2010.

26. Singh NP, Mccoy MT, Tice RR and Schneider EL: A simple technique for quantitation of low levels of DNA damage in individual cells. Exp Cell Res 175: 184-191, 1988.

27. Tang KF, Ren H, Cao J, Zeng GL, Xie J, Chen M, Wang L and He CX: Decreased dicer expression elicits DNA damage and up-regulation of MICA and MICB. J Cell Biol 182: 233-239, 2008.

28. Venkataraman GM, Suciu D, Groh V, Boss JM and Spies T: Promoter region architecture and transcriptional regulation of the genes for the MHC class I-Related chain a and b ligands of NKG2D. J Immunol 178: 961-969, 2007.

29. Bartek J, Bartkova J and Lukas J: DNA damage signalling guards against activated oncogenes and tumour progression. Oncogene 26: 7773-7779, 2007.

30. Gasser S, Orsulic S, Brown EJ and Raulet DH: The DNA damage pathway regulates innate immune system ligands of the NKG2D receptor. Nature 436: 1186-1190, 2005.

31. Ljunggren HG and Malmberg KJ: Prospects for the use of NK cells in immunotherapy of human cancer. Nat Rev Immunol 7: 329-339, 2007.

32. Liu J, Shen W, Tang Y, Zhou J, Li M, Zhu W, Yang H, Wu J, Zhang S and Cao J: Proteasome inhibitor MG132 enhances the antigrowth and antimetastasis effects of radiation in human nonsmall cell lung cancer cells. Tumour Biol 35: 7531-7539, 2014.

33. Zhang L, Tang H, Kou Y, Li R, Zheng Y, Wang Q, Zhou X and Jin L: MG132-mediated inhibition of the ubiquitin-proteasome pathway ameliorates cancer cachexia. J Cancer Res Clin Oncol 139: 1105-1115, 2013.

34. Winter M, Sombroek D, Dauth I, Moehlenbrink J, Scheuermann K, Crone $\mathrm{J}$ and Hofmann TG: Control of HIPK2 stability by ubiquitin ligase Siah-1 and checkpoint kinases ATM and ATR. Nat Cell Biol 10: 812-824, 2008.

This work is licensed under a Creative Commons Attribution-NonCommercial-NoDerivatives 4.0 International (CC BY-NC-ND 4.0) License. 